

\title{
La pénitence de saint Lambert à Stavelot (vers 675-682), une ordalie hagiographique?
}

\author{
Philippe $\operatorname{GEORGE}^{(*)}$ \\ Conservateur du Trésor de la Cathédrale de Liège
}

Vers 650, en Ardenne septentrionale, les monastères de Malmedy et de Stavelot furent constitués juxta regulam coenobiorum vel traditionem patrum. Les moines célèbrent régulièrement l'office et mènent, à l'abri des bruits et des dangers du monde, une vie retirée, toute entière consacrée à Dieu. Prier pour leurs bienfaiteurs, "la paix et la stabilité du royaume " sera la tâche principale assignée à « la congrégation de ceux qui chantent les louanges de Dieu ». Cette vision romantique des moines oblitère la gestion économique de leur domaine ${ }^{(1)}$, leurs intérêts profondément humains, et aussi ce que nous avons appelé " les armes monastiques ", alors que Stavelot-Malmedy offre des exemples particulièrement révélateurs de la violence au Moyen Âge ${ }^{(2)}$. Faut-il pour autant nier toute préoccupation spirituelle ? Les hagiographes ont bien sûr l'art d'embellir la vie de leurs héros et, au cours des siècles, l'extraordinaire entreprise bollandiste a progressivement séparé le bon grain de l'ivraie. Entre les lignes, il faut parvenir au plus près de la vérité historique et c'est difficile.

Un épisode particulièrement évocateur de la discipline de l'abbaye à ses origines est " la pénitence de la croix de saint Lambert à Stavelot »(3).

Après l'assassinat du roi Childéric II en 675, Lambert fut déposé et remplacé par l'évêque Pharamond; il fut exilé à Stavelot ${ }^{(4)}$. On a cru que Lambert était en butte à l'hostilité d'Ebroïn; or il semble que ce soit l'inverse qui est vrai : Lambert fut écarté de son siège épiscopal par

(*) Colomban, Benoît, Remacle, Wibald et les autres nous ont plus d'une fois rapprochés, Alain Dierkens et moi depuis les années 70 . Son amitié ne m’a jamais fait défaut, sans doute parce que Benoît recommande avant tout d'écouter. Je suis très heureux de lui offrir cette modeste contribution au sein de Mélanges qui marquent pour lui une nouvelle étape de vie que je lui souhaite exceptionnelle.

(1) Dans leur thèse respective, Alain Dierkens et, pour Stavelot-Malmedy, Nicolas Schroeder ont remarquablement exploré ces sujets.

(2) Par exemple dans le Catalogue de l'exposition Liège autour de l'an mil. La naissance d'une principauté, Liège, Éditions du Perron, 2000, ou dans notre article "Maledictio adversus ecclesiae Dei persecutores. À propos d'un ouvrage récent », dans Revue belge de philologie et d'Histoire, t. 73, 1995, 4, p. 1011-1017.

(3) Nous renverrons, sans en être pleinement satisfait, à notre notice article « StabloMalmedy » dans Reallexikon der Germanischen Altertumskunde, t. 29, Berlin, De Gruyter, 2005, p. 430-435.

(4) Ad monastirium qui vocatur Stabolaus (Vita Landiberti vetustissima, c. 5, éd. Bruno KRUSCH, dans MGH, SRM, t. 6, Hanovre, Hahn, 1913, p. 358. Extrait complet : http :/ www.dmgh.de/de/fs1/object/display/bsb00000753_00366.html?sortIndex $=010 \% 3 \mathrm{~A} 020 \% 3$ A $0006 \% 3 \mathrm{~A} 010 \% 3 \mathrm{~A} 00 \% 3 \mathrm{~A} 00$ [consulté le 8 février 2018]. 
Wulfoald et Dagobert II qui lui reprochaient peut-être ses accointances avec Ébroïn ${ }^{(5)}$. Ainsi entre 675 et 682 , au plus tôt, l'évêque séjourna en exil dans l'abbaye ardennaise, fondée par saint Remacle ${ }^{(6)}$. Des sept ans de cette vie d'humilité - in laboribus, in ieiuniis, in vigiliis, in orationibus, in caritate, in longanimitate -, le récit de la pénitence de la croix fut rapporté à l'auteur de la Vita Landiberti episcopi Traiectensis vetustissima par Théoduin, l'un des deux serviteurs de Lambert à Stavelot. Une nuit, prolongeant ses prières au dortoir, Lambert laissa tomber une de ses sandales sur le pavé et troubla ainsi le silence du monastère. L'abbé réveillé, ignorant quel était l'auteur du bruit, enjoignit au coupable d'aller immédiatement en pénitence à la croix : Qui istum sonum fecit, statim ambulet ad crucem! Que désigne vraiment cette expression? Près du monastère, selon Sigebert de Gembloux (vers 1111), entre l'oratoire et le dortoir, se dressait une croix en pierre. Lambert obtempéra aussitôt : il sortit de son lit et quitta le dortoir, pieds nus, sans sandales et vêtu d'un simple cilice, il se rendit à la croix et, inflexible, les bras tendus - immobilis persistens, protensis manibus, inflexibile brachia -, il récita des psaumes. Les moines se levaient au chant du coq, et au signal donné, ils entraient dans le sanctuaire pour les vigiles. À cause du froid, après l'office, ils se précipitaient au chauffoir. On était en effet au cœur de l'hiver et il neigeait abondamment. C'est à ce moment que l'abbé s'inquiéta de savoir si tous les frères étaient là et l'un d'eux lui fit remarquer qu'il avait envoyé Lambert à la croix. L'abbé se rendit compte de sa méprise et se confondit en excuses auprès de l'évêque. Celui-ci fut découvert frigorifié - semper stabat invictus ad crucem -, et il fallut le réconforter par un bain chaud.

L'interprétation de ce texte est difficile, par ses références possibles à différentes coutumes et sources ${ }^{(7)}$. Ad crucem [...] iussus se retrouve dans la Règle des chanoines de saint Chrodegang : le chapitre 17 prescrit que le clerc obstiné ou désobéissant, qui se rebellerait d'aller à la croix, serait sermonné par ses aînés (Regula, c. XXXIII, col. 1073). Nec mora relicto évoque la Règle de saint Benoît en son chapitre 5 sur l'obéissance : Primus humilitatis gradus est obodentia sine mora. Le premier degré d'humilité est l'obéissance sans délai. Dès que le supérieur a donné un ordre, on ne peut en différer l'exécution ${ }^{(8)}$. En son chapitre 42 «Que personne ne parle après complies », la Règle de saint Benoît rapporte que «Les moines doivent s'appliquer au silence en tout mais principalement pendant la nuit. [...] Au sortir de cette Heure [Complies], il ne sera plus permis à personne de dire quoi que ce soit. Si quelqu'un viole cette règle du silence, il sera puni rigoureusement

(5) Démonstration par Matthias WERNER, Der Lütticher Raum in frühkarolingischer Zeit, Göttingen, Vandenhoeck \& Ruprecht, 1980 (Veröffentlichungen des Max-PlanckInstituts für Geschichte, 62), p. 256-263.

(6) Saint Remacle, dont on ignore l'année du décès (un 3 septembre avant 677), aurait même pu connaître Lambert. Voir la bibliographie et l'état de la question dans Philippe GEORGE, "L'Aquitaine et le pays mosan. Sur les pas de saint Remacle », dans Edina BozóKy, éd., Saints d'Aquitaine. Missionnaires et pèlerins du haut Moyen Âge, Rennes, Presses universitaires de Rennes, 2011, p. 103-126.

(7) Comment oublier qu'en 1980 nous en avions discuté avec Dom Jacques Dubois dans le cadre de son cours d'hagiographie et d'histoire monastique aux Hautes-Études ?

(8) C'est d'ailleurs dans ce sens que Sigebert de Gembloux interprète ce passage auquel il donne pour titre : De obcedientia et humilitate sancti Lamberti, cf. éd. Bruno KRUSCH, dans $M G H, S R M$, t. 6, p. 396. 
[...] ». Au chapitre 60, à propos des « prêtres qui désireraient se fixer dans le monastère » $[\ldots]$, le candidat « $[. .$.$] saura qu'il sera tenu à toute la discipline$ de la Règle, et qu'on ne lui relâchera rien [...]. Il est soumis à l'observance régulière et qualifié plutôt pour donner à tous des exemples d'humilité ». La Regula coenobialis de Colomban prescrit qu'une pollution nocturne est punie par la récitation des psaumes ${ }^{(9)}$ et le second pénitentiel précise le nombre de psaumes : de 6 à 30, en passant par 12, 15 et 24. La Règle des moines de Colomban, en son chapitre 2 «Du silence », ne prescrit rien de particulier quant au silence à respecter au dortoir ${ }^{(10)}$, ni quant à la pratique de la pénitence de la croix ${ }^{(11)}$. Par contre, on trouve l'expression « se crucifier » sous la plume de quelques hagiographes irlandais, ce qui doit être une allusion à la même pratique de pénitence ${ }^{(12)}$. La «prière de la croix » (« crossfigell ») est prescrite dans les pénitentiels irlandais ${ }^{(13)}$. C'est l'habitude ancienne de prier les bras en croix, exercice pouvant devenir fatigant, qui est à l'origine de la pratique de mortification jointe précisément à la prière ${ }^{(14)}$. L'expression Iudicare crucem dans la Vita Landiberti n'évoque-t-elle pas le droit médiéval ? Difficile de s'y retrouver dans tous ces textes... Et si nous nous tournions vers l'iconographie ? Car ce séjour de saint Lambert à Stavelot va se perpétuer dans sa légende. Ne prenons comme exemple, bien en vue dans l'ancienne cathédrale de Liège, que la belle peinture de Bertholet Flémal $(\dagger 1675)$ qui ornait jadis un autel du jubé(15) (fig. 1).

(9) SAINT Colomban, Règles et pénitentiels monastiques, introduction, traduction et notes par Adalbert de VoGÜÉ dans IDEM, Aux sources du monachisme colombanien, t. 2, Abbaye de Bellefontaine, 1997 (Vie Monastique, 20), p. 76.

(10) Colomban, Règles et pénitentiels, p. 55.

(11) Contrairement à ce qui a été écrit jusqu'à présent, et notamment par Dom Louis Gougaud, Dévotions et pratiques ascétiques du Moyen Âge, Paris, Desclée De Brouwer, 1925 , p. 9, le Père de Vogüé ne croit pas que la Règle de saint Colomban prescrive la pratique de la pénitence de la croix (Regula Coenobalis, III, 5 [Patrologie Latine, c. X, col. 217] ; Colomban, Règles et pénitentiels, op. cit., p. $122=\mathrm{III}, 5$ et notes] ; il traduit l'expression crucem non adierit par celui qui ne s'approche pas de la croix, c'est-à-dire d'une croix plantée devant la maison. Même traduction chez George Stuart Murdoch Walkers, S. Columbani Opera, Dublin, Dublin Institute for Advanced Studies, 1957 (Scriptores latini Hibernie, 2), p. 146-149. Renseignements aimablement transmis par feu le Père de Vogüé.

(12) L. Gougaud, Dévotions, op. cit., avec bibliographie.

(13) Allen J. FrANTZEN, La littérature de la pénitence dans l'Angleterre anglosaxonne, Fribourg, Éditions universitaires, 1991 (Studia Friburgensia, nv. série, 75) et V.O'. MAIDIU, « Regole monastiche irlandesi » dans Dizionario degli Istituti di perfezione, t. VII, Rome, Edizioni Paoline, 1983, col. 1522-1533 : Règle de Ciaran (col. 1525-1526) et Règle de Tallaght (col. 1532), règles réunies et traduites par Eoin DE BHALDRAITHE, « Irish Monastic Rules », dans Hallel, XV, p. 47-92. Renseignements aimablement communiqués par feu le Père de Vogüé.

(14) Dans la tradition chrétienne, le souvenir de Moïse priant les bras étendus et celui de la crucifixion du Christ vont se fondre et de nombreux saints sont représentés dans cette attitude. Ainsi, saint Lambert mourut les bras en croix, de même saint Poppon de Stavelot $(\dagger 1048)$ - extensa brachia in cruce, extensis manibus -, ou saint Mengold de Huy, selon leur Vitae respectivement du $\mathrm{XI}^{\mathrm{e}}$ et du XII ${ }^{\mathrm{e}}$ siècle.

(15) Pierre-Yves KaIRIs, Bertholet Flémal (1614-1675). Le "Raphaël des Pays-Bas» au carrefour de Liège et de Paris, Paris, Arthéna, 2015, p. 148. 


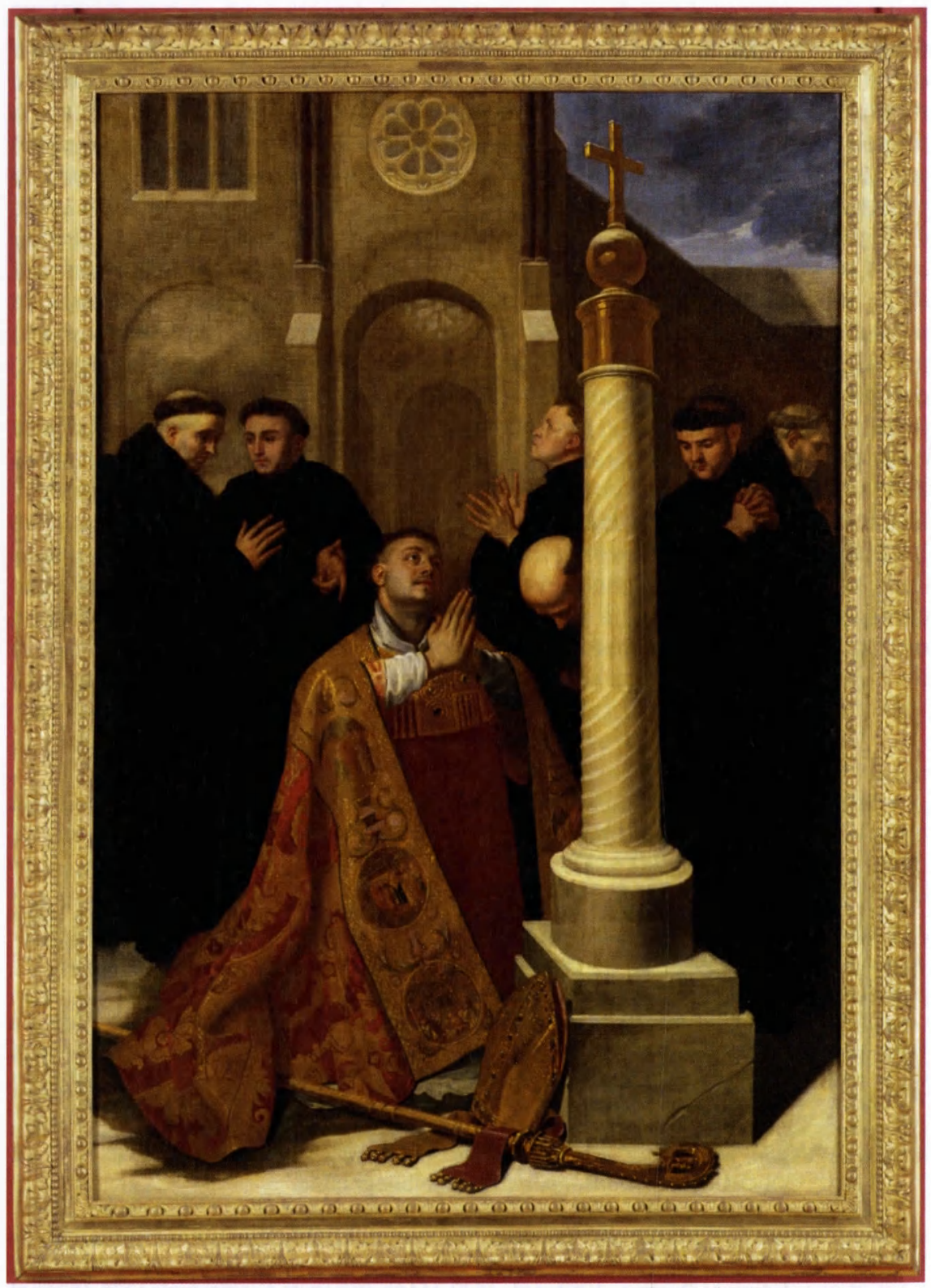

Fig. 1 : Ancienne cathédrale Saint-Lambert, Bertholet Flémal, avant 1675 C RMN 
C'est cette pénitence de Stavelot que représente, dans le retable de Lunebourg (Lüneburg, Basse-Saxe), Hans Bornemann, un peintre actif à Hambourg vers 1456-1458 (fig. 2). "Quand on embrasse d'un regard d'ensemble toutes les œuvres destinées à honorer saint Lambert, le retable de Lunebourg est une des plus attachantes ». Ainsi s'exprimait le Père Baudouin de Gaiffier dans un magistral article qu'il publia en $1973^{(16)}$ en complément à l'inventaire iconographique de saint Lambert réalisé par Mère Marie-Henri (Marguerite Bribosia) en 1955. Le bollandiste manifestait - à juste titre -, son admiration pour le travail de recherche de la chanoinesse, en expliquant avec la science et la modestie du savant comment l'œuvre avait pu échapper à la perspicacité de sa coreligionnaire ${ }^{(17)}$. C'est pour une église dédiée à saint Lambert que deux peintures furent commandées à Hans Bornemann. Mais la démolition de l'église en 1860 et le transfert du retable en l'église SaintNicolas de la même ville vinrent perturber la donne, d'autant plus qu'une restauration de 1868 inscrivit sur le nimbe « Saint Laurent » au lieu de " Saint Lambert ». Juxtaposées, les deux scènes forment la moitié droite dormante d'un retable, l'une montre la pénitence de Stavelot, l'autre le martyre de saint Lambert. Les autres scènes ont trait à la passion des apôtres Jude et Thaddée. Dans la scène qui nous intéresse, l'évêque auréolé, vêtu d'une longue bure mais coiffé d'une mitre, est suspendu à une croix. Plusieurs personnages l'entourent, dont sans doute le donateur, agenouillé, qui revêt les pieds de Lambert de chaussons. Bornemann ouvre la perspective sur un paysage où un monastère de Lunebourg remplace celui de Stavelot dans un environnement calme et verdoyant, manière d'actualiser la scène et son message. Deux phylactères instaurent un dialogue entre l'abbé et Lambert avec des phrases tirées de la Vita prima Landiberti, mais mal retranscrites par le peintre ${ }^{(18)}$. On est loin de Stavelot, loin des Bénédictins, loin de la neige qui, selon la Vita, tombait abondamment et avait quasi frigorifié l'évêque ; par contre c'est un témoignage iconographique précis sur le mode de pénitence de la croix. Mélanie Luck von Claparède a aussi raison de comparer Lambert crucifié au Volto santo de Lucques bien connu dans la région ${ }^{(19)}$. Quant à l'autre peinture, la scène du martyre, son caractère particulier réside dans la

(16) Baudouin de GAIFFIER, À propos de l'iconographie de saint Lambert, rééd. dans Baudouin de GAIFFIER, Recueil d'hagiographie, Bruxelles, Société des Bollandistes, 1977 (Subsidia hagiographica, $\mathrm{n}^{\circ} 61$ ), $\mathrm{n}^{\circ}$ XII.

(17) Depuis 1981 nous poursuivons cet inventaire iconographique dans le Bulletin de la Société royale Le Vieux-Liège, voir https ://orbi.uliege.be/browse?type=authorulg\&rpp $=20 \&$ value $=$ George $\% 2 C+$ Philippe + p001686 [consulté le 8 février 2018].

(18) Sur une banderole, se déployant de la bouche de l'abbé vers le haut, on lit : Ingnosce mihi, ingnosce quia nesciens peccavi; da in < dulgentiam > sur une autre banderole est inscrite la réponse de saint Lambert : $\langle$ D $>$ ata sit vobis a Deo indulgentia; in frigore, phrase correspondant aux textes de la Vita.

(19) Ralph HENNINGS \& Mélanie LUCK von CLAPARÈDE, Der heilige Lambertus und die Lambertikirche in Oldenburg, Oldenburg, Lamberti-Verlag, 2005. 


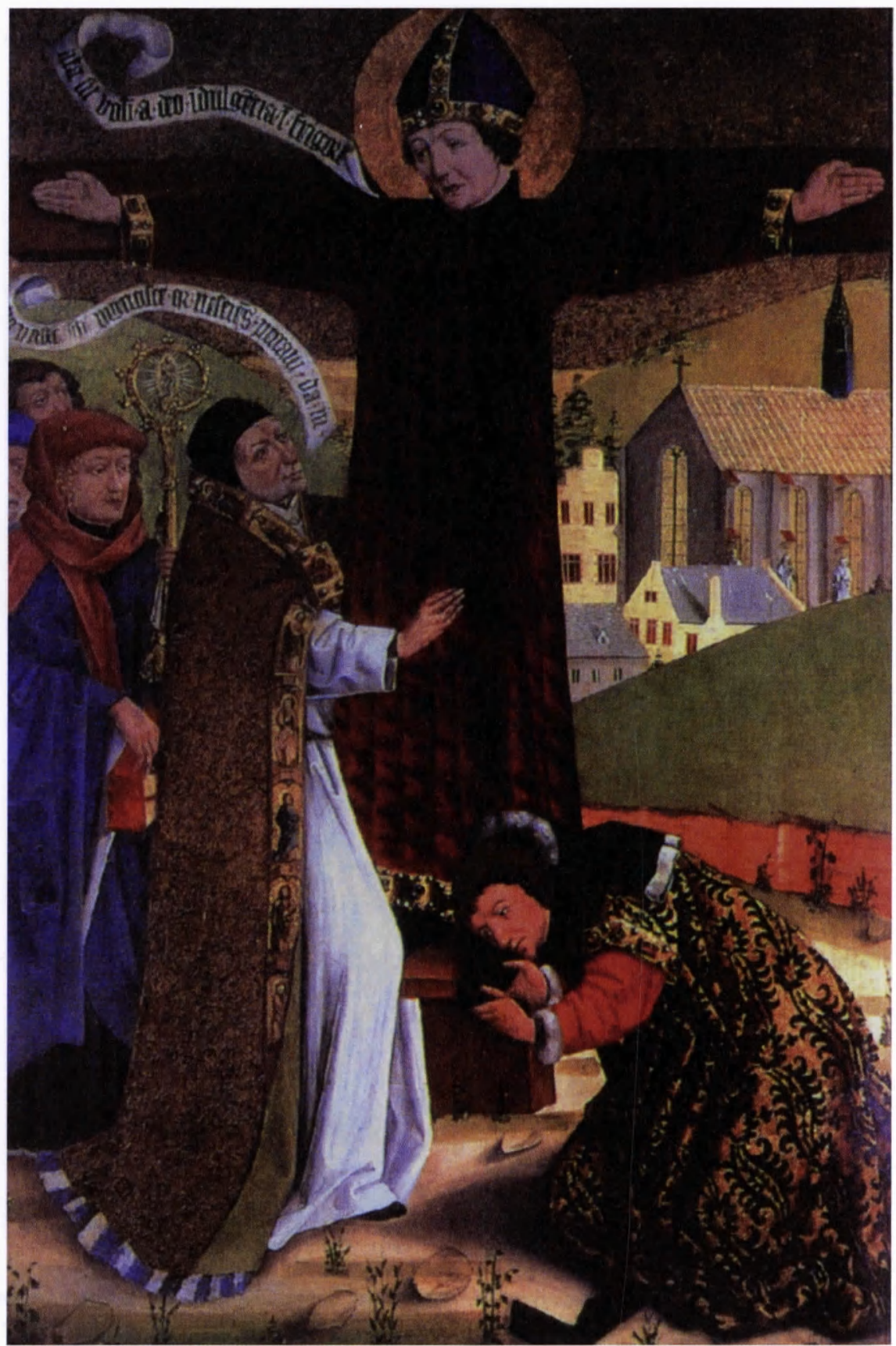

Fig. 2 : Lunebourg, église Saint-Nicolas, Hans Bornemann, vers 1456-1458 (C) Trésor 
présence d'une épée aux côtés de Lambert ${ }^{(20)}$; elle évoque, une fois encore à travers la Vita vetustissima ${ }^{(21)}$, le sursaut de défense qu'eut l'évêque à l'approche des meurtriers. Ce sursaut fut vite réfréné, et le saint de rejeter son épée et de se plonger dans la prière, assumant pleinement son martyre.

L'iconographie singulière de Lunebourg présente saint Lambert suspendu à une croix. Cela nous met à l'esprit que l'attitude des bras en croix fut employée comme ordalie au Moyen Âge ${ }^{(22)}$. Le contexte de la pénitence n'est pas sans rapport avec le contexte judiciaire du jugement de Dieu ${ }^{(23)}$. De ce point de vue, ne pourrait-on penser à une référence à l'ordalie iudicium crucis ? Mal connue de l'époque carolingienne, l'épreuve sera rapidement interdite $^{(24)}$. D'une ordalie permettant de savoir la vérité là où les procédures ordinaires échouent, l'épisode narré dans la Vita paraît bien offrir un point commun puisque Lambert survit à l'épreuve en dépit du froid glacial et qu'il en sort sain et sauf. Comment punir d'une faute et comment Lambert a-til vaincu la souffrance ? L'épreuve infligée et vaincue est déjà un moyen

(20) Cf. notre contribution "L'iconographie du meurtre de saint Lambert de Liège ", dans Natalie FRYDE \& Dirk REITZ, éds, Bischofsmord im Mittelalter. Murder of Bishops, Göttingen,Vandenhoeck \& Ruprecht, 2003 (Veröffentlichungen des Max-Planck-Instituts für Geschichte, 191), p. 171-185. Sur la peinture du martyre de Lunebourg par Hans Bornemann, la troupe est composée de deux personnages enturbannés. Une xylographie d'un passionnaire de 1488 en allemand, conservé à Trèves (Bibliothek des Priesterseminars), en est un peu le décalque inversé. Nous l'avons publiée dans nos « Iconographies de saints mosans, 16. Munich, Bayerische Staatsbibliothek, Cod. Gall. 3, $\mathrm{f}^{\circ} 182^{\mathrm{r}}$ Légendier français ca. 1430 "', dans Bulletin de la Société royale Le Vieux-Liège, t. 313, 2006, p. 45-48.

(21) Tunc sacerdus illico Landibertus, discalciatis pedibus, fortissimus præliator, continuo adprehenso gladio in manibus suis, ut contra hostes suos pugnaturus accederet. Ensuite, se fiant au Seigneur, il jeta son glaive gladio de manibus proiecit ad terram, préférant mourir que de se défendre.

(22) Bruno LemESLE, La main sous le fer rouge. Le jugement de Dieu au Moyen $\hat{A} g e$, Dijon, Éditions universitaires de Dijon, 2016, p. 25 : l'ordalie de la croix sous les Carolingiens «consistait à ce que les champions s'affrontent debout les bras en croix, face à face, de part et d'autre d'une croix. Le premier qui faiblissait avait perdu ». Nous remercions Bruno Lemesle qui nous a confirmé opportun le rapprochement que nous avons opéré.

(23) Le dossier aurait mérité davantage d'examen en sciences juridiques. On renverra à Jean GAUDEMET, "Les ordalies au Moyen Âge: doctrine, législation et pratique canoniques ", (1965) réimpr. dans IDEM, La société ecclésiastique dans l'occident médiéval, Londres, Variorum Reprints, 1980 (Collected Studies, 116), p. XV, 99-135. L'ordalie de la croix, instituée par les premiers carolingiens, consistait pour les personnes impliquées à se placer en forme de croix, être ligotées à un poteau et réussir à tenir le plus longtemps les bras levés à l'horizontale. Le premier à baisser les bras abandonnait, d'où l'expression "baisser les bras ". Louis le Pieux prohiba cette épreuve vers 819, l'accusant de parodier la Passion du Christ : Dominique BARTHÉLEMY, «Diversité des ordalies médiévales " dans Revue historique, t. 280, 1988, p. 3-25, et IDEM, "Ordalie ", dans Claude Gauvard, Michel Zink \& Alain de Libera, éds, Dictionnaire du Moyen $\hat{A} g e$, Paris, Presses universitaires de France, $2^{\mathrm{e}}$ éd., 2006, p. 1020-1022.

(24) François-Louis GANSHOF, "Les réformes judiciaires de Louis le Pieux » dans Comptes rendus des séances de l'Académie des Inscriptions et Belles-Lettres, $109^{\mathrm{e}}$ année, 1965, 2,p. 418-427 : www.persee.fr/doc/crai_0065-0536_1965_num_109_2_11893 [consulté le 8 février 2018] et Julien MAQUET, "Faire justice " dans le diocèse de Liège au Moyen Age (VIII $-X I I^{e}$ siècles) Essai de droit judiciaire reconstitué, Genève, Droz, 2008 (Bibliothèque de la Faculté de Philosophie et Lettres de l'Université de Liège, 290), p. 548-549. 
d'atteindre la sainteté(25), une anticipation de son martyre, apothéose hagiographique.

Notre titre accrocheur permet d'attirer l'attention sur l'interdisciplinarité, où iconographie et hagiographie ont leur place. Au cours des siècles la pratique pénitentielle de Stavelot est en tout cas mal comprise et l'on a vu plus haut toutes les réminiscences charriées par la tradition monastique et plus largement par la tradition médiévale.

Cela vaut pour le texte, mais il serait peut-être exagéré d'aller plus loin et de penser, dans le cas de cette peinture de Lunebourg, à une réminiscence de l'ordalie de la croix dont la mention n'est qu'éphémère sous Louis le Pieux. Pourtant l'ordalie unilatérale fut réinterprétée après le quatrième concile de Latran IV (1215) et notamment en iconographie jusqu'au $\mathrm{XV}^{\mathrm{e}}$ siècle $^{(26)}$.

Comment ne pas en même temps penser à une ordalie de sainteté avec le miracle du feu de la légende de saint Lambert, miracle de la jeunesse du saint illustré lui aussi abondamment et notamment sur son buste-reliquaire : Lambert enfant apporte du feu dans son surplis sans se brûler ${ }^{(27)}$. Cette nouvelle anticipation de la sainteté de l'évêque prend ainsi aussi une autre dimension ${ }^{(28)}$.

Pour revenir à la pénitence de Stavelot, à Stavelot même les reliques historiques gardent mémoire, au moins depuis le $\mathrm{XI}^{\mathrm{e}}$ siècle, des sandales de Lambert et/ou de Remacle : de scandaliis beati Lanb(er)ti sive Remagli c(on) fessoris $^{(29)}$. L'allusion est claire: les sandales que Lambert laissa tomber pendant la nuit. Parmi les reliques historiques de l'ancienne cathédrale SaintLambert de Liège, on trouve une croix de fer embellie de cuivre doré et de cristalle de roche posée sur la porte de la sacristie derrier le grand autel, et (illecqs emplombée) laquelle selon la remarque du répertoir de l'an 1665 est celle-là devant laquelle saint Lambert fut trouvé priant Dieu la nuit entouré de neige à Stavelot, suivant l'histoire ${ }^{(30)}$. On le voit, dans la cathédrale Saint-Lambert elle-même, haut-lieu du culte du saint patron du diocèse, la

(25) Huguette LEgros, «Quand les jugements de Dieu deviennent artifices littéraires ou la profanité impunie d'une poétique ", dans La justice au Moyen Age : Sanction ou impunité ? [en ligne], Aix-en-Provence, Presses universitaires de Provence, 1986 [généré le 08 février 2018]: http ://books.openedition.org/pup/3023?lang=frhttp ://books.open edition.org/pup/3023?lang=fr.

(26) Julie JouRDAN, "Ordalie, image et sermon après le concile de Latran IV ", dans Cahiers de Recherches médiévales et humanistes [En ligne], 25, 2013 [consulté le 08 février 2018] : http ://journals.openedition.org/crm/13111.

(27) François BOUGARD, « Le feu de la justice et le feu de l'épreuve, IV ${ }^{\mathrm{e}}$-XII ${ }^{\mathrm{e}}$ siècle ", dans Il fuoco nell' alto medioevo, Spolète, Centro italiano di studi sull' altomedioevo, 2013 (Settimane di studio della Fondazione del Centro italiano di studi sull' altomedioevo, $60)$, p. $389-436$.

(28) Alain DierKens, "Réflexions sur le miracle au haut Moyen Âge », dans Miracles, prodiges et merveilles au Moyen Age. Actes du XXV Congrès de la Société des Historiens médiévistes de l'Enseignement public (Orléans, 3-5 juin 1994), Paris, Publications de la Sorbonne, 1995, p. 9-30.

(29) Notre ouvrage Les reliques de Stavelot-Malmedy, Malmedy, Art et Histoire, 1989 , p. 50 , n. 17.

(30) Notre article « Le trésor des reliques de la cathédrale Saint-Lambert de Liège ", dans Bulletin de l'Institut archéologique liégeois, t. 117, 2013, p. 63-141. 
pénitence de Stavelot garde une place importante : reliques historiques au trésor, toile de Flémal à l'autel impérial du jubé mais aussi et surtout la magnifique représentation dans une des niches du socle au centre de son célèbre buste-reliquaire (vers 1512), sans doute l'œuvre la plus ostentatoire de la légende du saint (fig. 3$)^{(31)}$.

(31) La technologie permet à Thierry Lechanteur d'en appréhender tous les détails : https ://twitter.com/LechanteurT/status/962379320755937282 [Consulté le 10 février 2018]. 


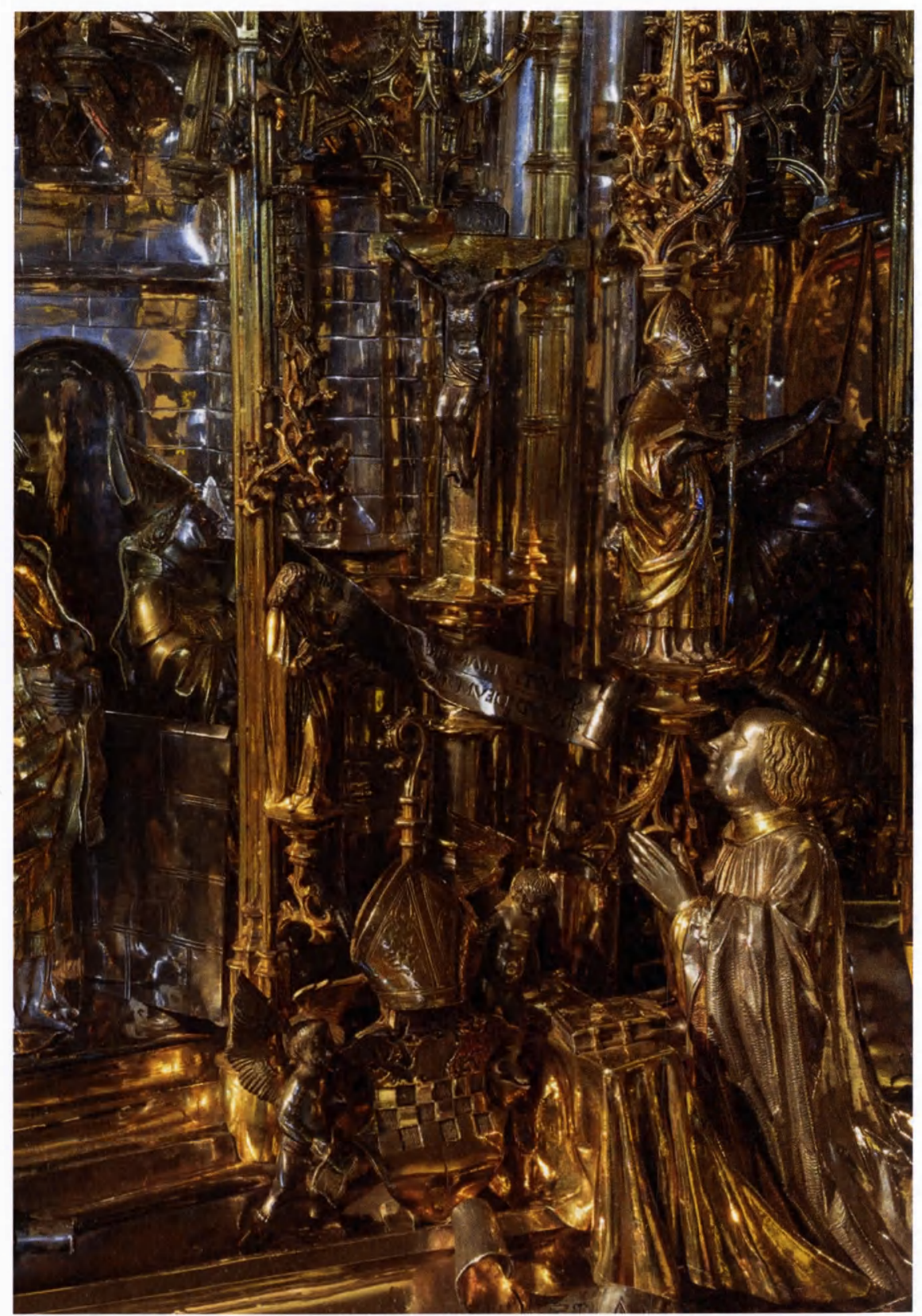

Fig. 3 : Trésor de Liège, buste-reliquaire de saint Lambert, vers 1512 C Trésor 


\section{RÉSUMÉ}

Philippe GeORge, La pénitence de saint Lambert à Stavelot (vers 675-682), une ordalie hagiographique?

La pénitence de saint Lambert ( $\dagger$ vers 696-705) au monastère de Stavelot est un épisode de la légende du saint patron du diocèse de Tongres-Maastricht-Liège, rapporté par la Vita antiquissima Landiberti, contemporaine des événements. Ce témoignage peut être confronté à la tradition monastique et juridique mais aussi à l'iconographie, pour chercher à approcher au plus près la vérité historique. Les reliques apportent quant à elles un témoignage plus concret encore du châtiment imposé à l'évêque en exil.

Saint Lambert - Stavelot - pénitence - iconographie - ordalie

\section{SUMMARY}

Philippe GeOrge, The Penance of St. Lambert in Stavelot (About 675-682) a Hagiographic Ordeal?

The penance of St. Lambert († c. 696-705) in the monastery of Stavelot is an episode of the legend of the patron saint of the diocese of Tongeren-Maastricht-Liege, reported by the Vita antiquissima Landiberti, contemporary of the events. This testimony can be confronted with the monastic and juridical tradition but also with the iconography in order to get as close as possible to the historical truth. The relics bring a more concrete testimony of the punishment imposed on the bishop in exile.

Saint Lambert - Stavelot - penance - iconography - ordeal

\section{SAMENVATTING}

Philippe GeORGE, De boetedoening van St. Lambertus in Stavelot (rond 675682) een hagiografisch godsoordeel?

De boetedoening van de H. Lambertus († 696-705) in het klooster van Stavelot is een episode van de legende van de patroonheilige van het bisdom Tongeren-MaastrichtLuik, zoals beschreven in de Vita antiquissima Landiberti, een tekst uit die tijd. Deze getuigenis kan worden geconfronteerd met de monastieke en juridische traditie, maar ook met de iconografie, om dichter bij de historische waarheid te komen. De relikwieën brengen een meer concrete getuigenis van de straf die de bisschop in ballingschap werd opgelegd.

Sint Lambertus - Stavelot - boetedoening - iconografie - beproeving 